NASZA DERMATOLOGIA Onlin OUR DERMATOLOGY Online

Source of Support: Nil

Competing Interests: None

\section{A STUDY ON CONTACT DERMATITIS TO HAIR DYE AND HENNA}

\author{
Neerja Puri, Asha Puri \\ Department of Dermatology and Venereology, Punjab Health Systems Corporation, \\ Ferozepur, Punjab, India
}

Corresponding author: Dr. Neerja Puri

neerjaashu@rediffmail.com

\begin{abstract}
P- Phenylenediamine is an oxidative chemical that is frequently used as a permanent hair-coloring agent. It is added to henna to increase the intensity and longevity of the tattoo and expedites its drying time. Henna itself is a greenish brown vegetable coloring made from the leaves of Lawsonia inermis and rarely causes allergic contact dermatitis. The addition of PPD causes the contact sensitization to black henna. Serious adverse skin reactions to permanent hair dyes and temporary black tattoos have been reported. As temporary tattoos have become fashionable among adolescents, the risk profile for p-phenylenediamine (PPD) sensitization of the population has changed simultaneously with an increasing use of hair dyes in this age group. With increased popularity of body art such as body piercing and tattooing, an increase in temporary henna tattoos has also occurred. Although the appeal of non-permanence exists for henna tattoos, dermatologists have begun to see numerous cases of allergic contact dermatitis linked with a certain type of henna. We selected 50 patients using hair dye and henna for our study. Patch testing was done in all the patients using Indian standard series of antigens. Regarding to the side effects to hair dye and henna and itching was the commonest symptom seen in $16 \%$ patients, erythematous scaly plaques were seen in $10 \%$ patients, vesicular reactions were seen in $6 \%$ patients, angioneurotic oedema and contact urticaria was seen in $4 \%$ patients each and anaphylaxis and keloidal reaction was seen in $2 \%$ patients each.
\end{abstract}

Key words: dermatitis; hair dye; henna; allergic; PPD

\section{Introduction}

Paraphenylenediamine is a chemical substance that is widely used as a permanent hair dye. Henna has been used for centuries in certain cultures as body paint and more recently as a hair coloring agent. It has been noted to be a very low-level sensitizer [1]. At the same time, body tattooing has enjoyed increased acceptance among the youth of many cultures, including those in the West. The active ingredient of henna is lawsone (2-hydroxy-1, 4-naphthoquinone) [2]. It is traditionally used in Islamic and Hindu cultures as a hair coloring and as a dye for decorating the nails or making temporary skin tattoos. Actually, henna has a very low allergic potential. In most cases, allergic reactions not caused by henna, but by the chemical coloring additives that are added to henna mixtures. A recent phenomenon is ,temporary tattooing," in which the tattoo colors the stratum corneum and lasts until that layer is shed. Such tattoos are usually referred to as henna tattoos or black henna tattoos. Recently, reports of reaction to these temporary henna tattoos have become common [3]. In the vast majority of such cases, the offending allergen has been found to be PPD. The level of PPD in these products is much higher than that found in hair color [4,5]. Because of PPD's high sensitization potential, the application of PPD to the skin is not an approved use. Since sensitization to PPD from tattoos is likely to be lifelong, we will likely see a population of individuals who will respond adversely to their attempts at hair coloring as they age [6]. Allergy to natural henna is not usual; however the addition of para-phenylenediamine (PPD) to the natural henna increases the risk of allergic contact dermatitis [7,8]. The objectives of the study were to identify the presence and concentration of PPD. PPD is present in concentration higher than that recommended for hair dyes in most of the black henna samples. The presence of PPD in the black henna increases the risk of allergic contact dermatitis among users of black henna $[9,10]$.

In the Arab world and Indian subcontinent henna is used for skin decoration and hair dying during social celebrations, and during marriage ceremonies people celebrate by adorning the bride, and sometimes the groom, with henna. Despite the wide spread use of natural henna, specially, in countries where henna art is traditionally practiced, reports of allergic contact dermatitis to natural henna are very rare in the literature. It can therefore be assumed that natural henna is a very weak skin allergen. 


\section{Materials and Methods}

We selected 50 patients using hair dye and henna for our study. Patch testing was done in all the patients using Indian standard series of antigens by CODFI (Contact Dermatitis Forum of India). Informed consent was taken from all the patients before the study. Prior approval of hospital ethical was taken. The patients were instructed to stop any oral corticosteroids before the patch testing. The patch testing reading was taken after 48 hours and 72 hours. Various types of reactions were noted and tabulated.

\section{Aims}

1. To study the various types of reactions to hair dye and black henna tattooes.

2. To see the patch testing positivity in all the patients.

\section{Results}

The data was tabulated and the results were analyzed statistically (Tabl I, II).

\begin{tabular}{|l|l|l|l|}
\hline \multicolumn{1}{|c|}{ Sr no } & \multicolumn{1}{|c|}{ Side Effects } & Number of patients & Percentage \\
\hline 1. & Angioneurotic oedema & 2 & 4 \\
\hline 2. & Itching & 8 & 16 \\
\hline 3. & Contact urticaria & 2 & 4 \\
\hline 4. & Vesicular reactions & 3 & 6 \\
\hline 5. & Erythematous scaly plaques & 5 & 10 \\
\hline 6. & Anaphylaxis & 1 & 2 \\
\hline 7. & Keloidal reaction & 2 & 4 \\
\hline
\end{tabular}

Table I. Side effects.

\begin{tabular}{|l|l|l|l|}
\hline \multicolumn{1}{|c|}{ Sr no } & \multicolumn{1}{|c|}{ Site } & Number of patients & Percentage \\
\hline 1. & eyelids & 5 & 10 \\
\hline 2. & ears & 2 & 4 \\
\hline 3. & forehead & 3 & 6 \\
\hline 4. & neck & 2 & 4 \\
\hline 5. & beard & 4 & 8 \\
\hline
\end{tabular}

Table II. Sites of involvement.

\section{Discussion}

The mean age group of patients in our study was $36+20$ years. Females outnumbered females and female: male was 1.5:1. The mean age at first hair dyeing was 25 years. Out of all the patients , 4\% patients had history of temporary tattoos during the childhood. Regarding to the side effects to hair dye and henna and itching was the commonest symptom seen in $16 \%$ patients, erythematous scaly plaques (Fig. 1) were seen in 10\% patients, vesicular reactions were seen in $6 \%$ patients, angioneurotic oedema and contact urticaria was seen in $4 \%$ patients each and anaphylaxis and keloidal reaction (Fig. 2) was seen in $2 \%$ patients each. The commonest site of involvement was eyelids seen in $10 \%$ patients, beard involvement was seen in $8 \%$ patients, forehead in $6 \%$ patients and neck and ear involvement was seen in $4 \%$ patients each. Ptch testing positivity was seen in $84 \%$ patients.

In recent years, attention has been drawn to the use of p-phenylenediamine (PPD) in henna dyes and the potential for this allergenic chemical to cause hyper sensitivity reactions [11]. It is the immediate, partially oxidized state that may cause allergy in sensitive individuals. Fully oxidized PPD is not a sensitizer [12]. Reaction is caused by the use of hair dye in mild cases only involves dermatitis to the upper eyelids or the rims of the ears. In more severe cases, it often extends beyond the scalp to include the forehead, neck, eyelids and face. It manifests as pruritic, oedematous, erythematous scaly patches and plaques and vesicular lesions in some cases. Severe allergy to PPD can result in contact urticaria and rarely anaphylaxis. Dermatitis on the hands is seen in hair dressers. Other skin reactions, such as post inflammatory hyper or hypopigmentation, can also ensue. There have been rare reports of sensitivity occurrence with ordinary henna tattoos; however, henna containing paraphenylene diamine, a popular chemical for use in black henna to darken the tattoo and reduce fixation time, increases skin sensitivities $[13,14]$.

Short-term exposure to high levels of PPD (acute effects) may cause severe dermatitis, eye irritation and tearing, asthma, gastritis, renal failure, vertigo, tremors, convulsions, and coma in humans. Recently para-phenylenediamine has been mixed with natural henna to give an ebony color (black henna) instead of the orange/reddish color given by natural henna. The other reason for adding PPD to the natural henna is to speed up (shorten the time) of the tattooing process, while natural henna staining takes 4 to 12 hours, addition of PPD can reduce this time to an hour or two and also there will be a longer lasting effect as well. Thus, a new pattern of exposure to PPD has been recognized through henna art which increases the risk of developing adverse health effects related to PPD [15]. 
Acute allergic contact dermatitis, eczema, chemical burn, acute renal failure, acute and severe angioneurotic edema, abdominal pain and vomiting as adverse health effects associated with the use of henna containing PPD (black henna) are well documented in the literature [16]. In addition, people developeing sensitization from use of black henna are susceptible to cross reaction to oxidative hair dyes and to clothing dyes.

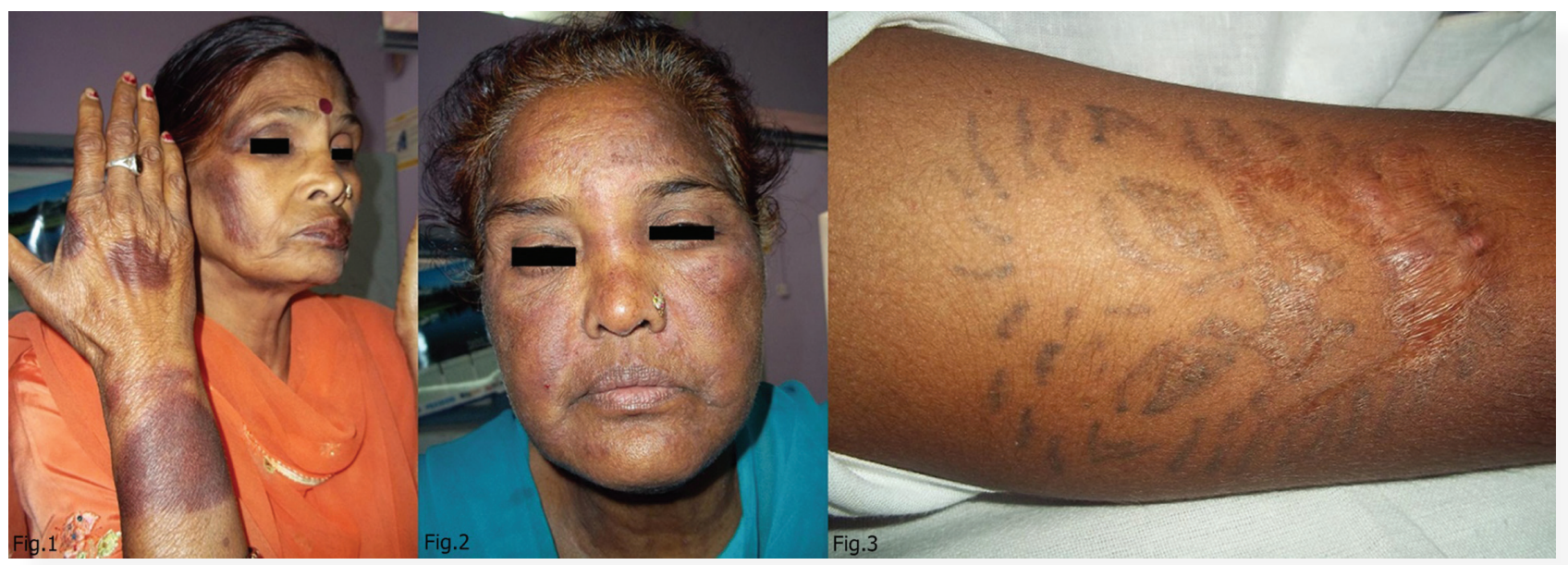

Figure 1. Severe allergic reaction to black henna in a 48 year old female.

Figure 2. Severe keloidal reaction to a black henna tattoo in a 20 year old male.

Figure 3. Keloidal reaction to black dye.

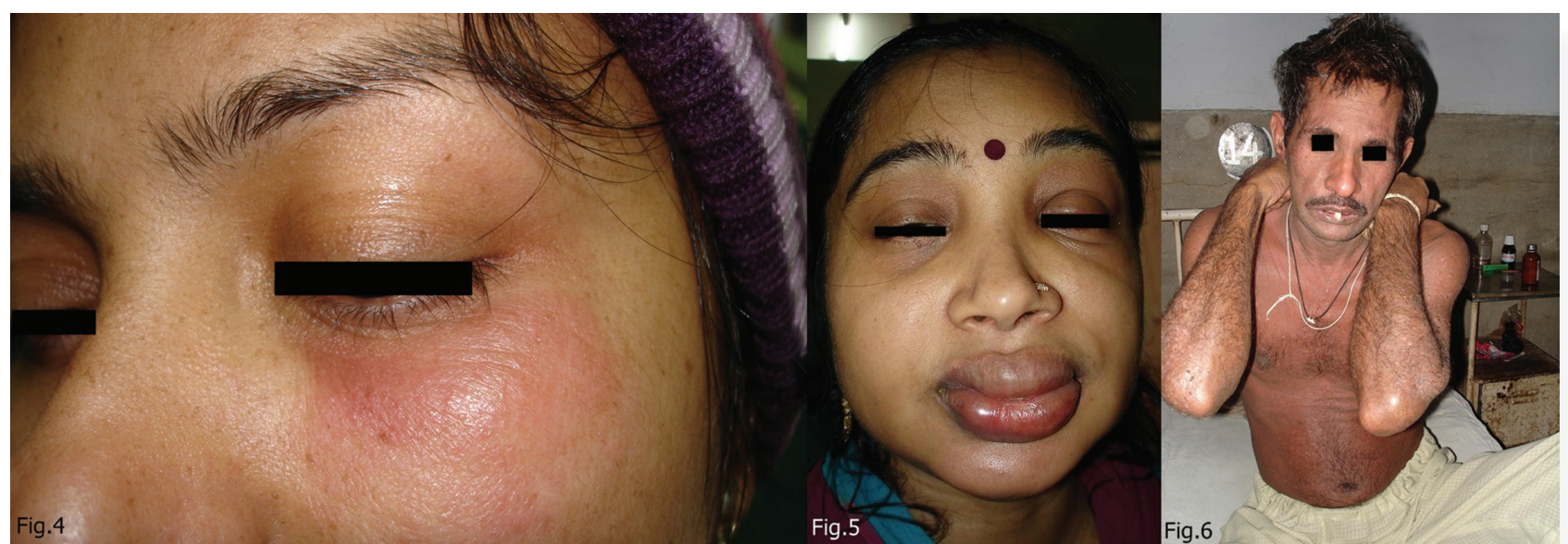

Figure 4. Erythematous scaly plaques in a 37 years old female.

Figure 5. Angioneurotic oedema in a 30 years old female after application of khadi black henna.

Figure 6. Erythrodermic reaction in a 53 years old man after applying hair dye.

\section{Conclusion}

The rate of adverse allergic skin reactions to hair dyes was higher than expected from patch test studies. Only by studying the clinical types of adverse reactions to hair dyes will it be possible to gather a complete epidemiological picture of the nature and extent of the problems related to hair dye ingredients.

\section{REFERENCES}

1. Pasricha JS, Grupta R, Panjwani S: Contact dermatitis to henna (Lawsonia) Contact Dermatitis. 1980;6:288-9.

2. Thami GP, Kaur S, Kanwar AJ: Allergic contact dermatitis to henna. Allergy. 2001; 56: 1013-4.

3. Wolf R, Wolf D, Matz H, Orion E: Cutaneous reactions to temporary tattoos. Dermatol Online J. 2003;9:3.
4. Matulich J, Sullivan J: A temporary henna tattoo causing hair and clothing dye allergy. Contact Dermatitis. 2005;53:33-6.

5. Kang IK, Lee MH: Quantification of para-phenylenediamine and heavy metals in henna dye. Contact Dermatitis. 2006;55:26-9.

6. Jovanovic DL, Slavkovic-Jovanovic MR: Allergic contact dermatitis from temporary henna tattoo. J. Dermatol. 2009;36:63-5.

7. Brancaccio RR, Brown LH, Chang YC, Fogelman JP, Mafong EA, Cohen DE: Identification and Quantification of paraPhenylenediamine in a Temprorary Black Henna Tattoo. Am J Contact Dermat. 2002;13:15-8.

8. Zapolanski T, Jacob SE: Para-Phenylenediamine. Dermatitis. 2008;19:20-1.

9. Schnuch A, Lessmann H, Frosch PJ, Uter W: ParaPhenylenediamine: the profile of an important allergen. Results of the IVDK. Br J Dermatol. 2008;159:379-86. 
10. Di Prisco MC, Puig L, Alomar A: Contact dermatitis due to paraphenylenediamine (PPD) on a temporal tattoo with henna: Cross reaction to azoic dyes. Invest Clin. 2006;47:295-9.

11. Nawaf AM, Joshi A, Nour-Eldin O: Acute allergic contact dermatitis due to para-phenylenediamine after temporary henna painting. J Dermatol. 2003;30:797-800.

12. Neri I, Guareschi E, Savoia F, PatriziA: Childhood Allergic Contact Dermatitis from Henna Tattoo. Pediat Dermatol. 2002;19:503-5.

13. Deleo VA: P-Phenylenediamine. Dermatitis. 2006;17:53-5.
14. Redlick F, Dekoven J: Allergic contact dermatitis to paraphenylenediamine in hair dye after sensitization from black henna tattoos: a report of 6 cases. Can Med Assn J. 2007;176:445-6. 15. Belhadjali H, Ghannouchi N, Amri Ch, Youssef M, Amri M, Zili $\mathrm{J}$ : Contact dermatitis to henna used as a hair dye. Contact Dermatitis. 2008;58:182.

16. Oztaş MO, Onder M, Oztaş P, Atahan C: Contact allergy to henna. J Eur Acad Dermatol Venereol. 2001;15:91-2.

Copyright by Neerja Puri, et al. This is an open access article distributed under the terms of the Creative Commons Attribution License, which permits unrestricted use, distribution, and reproduction in any medium, provided the original author and source are credited. 